\title{
BMJ Open Lipodystrophy among patients with HIV infection on antiretroviral therapy: a systematic review protocol
}

\author{
Lorena Gomes Cunha Lana, ${ }^{1}$ Daniela Rezende Garcia Junqueira, ${ }^{1,2,3}$ \\ Edson Perini, ${ }^{1}$ Cristiane Menezes de Pádua ${ }^{1}$
}

To cite: Lana LGC, Junqueira DRG, Perini E, et al. Lipodystrophy among patients with HIV infection on antiretroviral therapy: a systematic review protocol. BMJ Open 2014;4:e004088. doi:10.1136/bmjopen-2013004088

- Prepublication history for this paper is available online To view these files please visit the journal online (http://dx.doi.org/10.1136/ bmjopen-2013-004088)

Received 24 September 2013 Revised 3 February 2014 Accepted 10 February 2014

\section{(a) CrossMark}

${ }^{1}$ Departamento de Farmácia Social, Faculdade de Farmácia, Centro de Estudos do Medicamento (Cemed), Universidade Federal de Minas Gerais (UFMG), Belo Horizonte, Minas Gerais, Brazil ${ }^{2}$ Faculty of Health Sciences, The University of Sydney, Sydney, Australia ${ }^{3}$ Programa de Pós-graduação em Ciências Farmacêuticas, Universidade de SorocabaUniso, Sorocaba, São Paulo, Brazil

\section{Correspondence to}

Professor Cristiane Menezes de Pádua;

campadua@farmacia.ufmg.br

\section{ABSTRACT}

Introduction: Lipodystrophy is a frequent and disfiguring adverse effect of antiretroviral therapy (ART) in patients with HIV. It affects the quality of life of the patient and adherence to treatment, and generates new needs for comprehensive healthcare services. The aim of this study will be to conduct a systematic review of the literature from observational studies and describe lipodystrophy among patients with HIV infection during current or previous use of ART.

Methods and analysis: A systematic review of observational studies published in MEDLINE, CINAHL, LILACS, EMBASE and International Pharmaceutical Abstracts will be carried out. Citations of included studies will be checked to identify additional studies not identified in the electronic searches. It will include any observational study that considered lipodystrophy as the primary or secondary outcome and that had enrolled adolescent and adult patients with HIV infection who were on current or previous ART for at least 6 months. Data extraction and analysis will be performed independently by two reviewers. The extracted data will be discussed, decisions documented and, where necessary, the authors of the studies will be contacted for clarification. Measures of frequency, prevalence and incidence of lipodystrophy will be stratified according to definition, method of diagnosis and risk factors of the outcome.

Ethics and dissemination: Ethics is not required given this is a protocol for a systematic review. The findings of this study will be widely disseminated through peer-reviewed publications and conference presentations. Updates of the review will be conducted to inform and guide healthcare practice.

Protocol registration: PROSPERO—42013005450.

\section{INTRODUCTION}

The benefits achieved with the highly active antiretroviral therapy (HAART), introduced in the late 1990s for the treatment of HIV infection, are unquestionable. The decrease in AIDS mortality has been attributed mainly to the reduction in AIDS-related comorbidity and opportunistic infections. However, the increase in non-AIDS-related diagnoses, such as adverse reactions to antiretrovirals, has afforded HIV infection the characteristic of a chronic degenerative disease. ${ }^{1-3}$

At the beginning of antiretroviral treatment, adverse events tend to be common and of mild to moderate relevance. ${ }^{4}$ During the treatment course, adverse events become less frequent. However, late adverse events are more complex and lead to stigmatising conditions, affecting the quality of life of patients and their adherence to antiretroviral therapy (ART) ${ }^{56}$ thus generating new needs for comprehensive healthcare services. ${ }^{7}$

Morphological alterations caused by abnormalities in body fat distribution (lipodystrophy) are caused by the interaction of multiple factors such as ART, genetic predisposition of the patient, environmental factors and HIV infection. ${ }^{8}{ }^{9}$ However, epidemiological studies ${ }^{10}{ }^{11}$ have demonstrated a strong causal relationship between ART and lipodystrophy. Thus, lipodystrophy is considered an adverse effect of ART, and it is a public health issue of great relevance, since it is associated with insulin resistance, diabetes mellitus and dyslipidaemia-known risk factors for cardiovascular disease. ${ }^{12}$ Lipodystrophy is characterised by fat accumulation (hypertrophy) in one or more anatomical sites (eg, abdomen, dorsocervical spine, breasts) or fat loss (atrophy) mainly on the face, buttocks and extremities or mixed lipodystrophy (combination of lipoatrophy and lipohypertrophy). ${ }^{13}$ The long-term use of ART, the use of regimens containing a nucleoside analogue reverse transcriptase inhibitor (particularly stavudine) and a protease inhibitor, older age, gender and duration of HIV infection have been described as the main risk factors for lipodystrophy. ${ }^{12} 13$

The number of published observational studies of lipodystrophy has grown markedly in the past few years. Studies indicate that 
the prevalence of lipodystrophy in patients with HIV infection on ART ranges from $11 \%$ to $83 \% .{ }^{14}$ Nevertheless, these findings are derived from different definitions of lipodystrophy, as well as distinct selection criteria and follow-up of the study population. Owing to this variability, it is important to stratify the information available about estimates of morbidity and risk factors of lipodystrophy in regard to the different methods and criteria diagnosis employed, population characteristics and types of study designs. A systematic review of published studies may assist in clinical decisions and in public health actions, contributing to the best adherence to ART. In addition, this study will contribute to the improvement of a standardised definition for this important adverse drug reaction which significantly impacts treatment and quality of life of patients who are HIV positive.

\section{OBJECTIVES}

Our aim is to describe lipodystrophy among patients with HIV infection in the current or previous use of ART through the development of a systematic review of observational studies.

\section{METHODS AND ANALYSIS}

This will be a systematic review focused on an adverse drug reaction of ART. Data collection, analysis, presentation and interpretation of results will be performed based on standard guidelines for systematic review of adverse effect of healthcare interventions. ${ }^{15} 16$

\section{Criteria for considering studies for this review \\ Types of studies}

Observational studies irrespective of language and publication status, comparing patients with HIV infection on different antiretroviral regimens irrespective of the number of participants in each arm.

\section{Types of participants}

Adolescent and adult patients with HIV infection on current or previous ART use for at least 6 months.

\section{Types of interventions}

Observational studies investigating the occurrence of lipodystrophy as the primary or secondary outcome.

\section{Types of outcome measures}

The diagnosis of lipodystrophy performed by clinical evaluation and/or by self-report of patient, confirmed or not by other techniques, characterised by at least one of the alterations as follows. ${ }^{13}$

Lipohypertrophy

- Abdominal obesity;

- Breast hypertrophy in women;

- Increased fat side of the neck;

- Increased fat back of the neck;

- Dorsocervical and suprapubic lipomas.
Lipoatrophy

- Fat loss in the face;

- Fat loss in the buttocks;

- Fat loss from legs;

- Fat loss in the arms;

- Disclosure of veins in the muscles of the upper and lower limbs.

Mixed lipodystrophy

- Combination of lipoatrophy and lipohypertrophy.

\section{Search methods for identification of studies}

\section{Electronic searches}

A computerised search will be conducted of MEDLINE, CINAHL, LILACS, EMBASE and International Pharmaceutical Abstracts, using a search strategy combining free terms and indexing terms. The search strategy can be found in box 1 .

\section{Searching other resources}

Citations of included studies will be checked to identify additional studies not identified in the electronic search.

\section{Data collection and analysis}

\section{Selection of studies}

The selection of articles to be assessed in this review includes two steps. In the first stage, information from titles and abstracts will be screened for exclusion of nonrelevant retrieved papers. The potentially relevant articles will be read in full by two independent reviewers and cases of disagreement will be resolved by consensus. The selection process will be carried out according to the criteria for inclusion mentioned previously.

\section{Data extraction and management}

Two reviewers will independently extract data from included trials and cases of disagreement will be resolved by consensus. Extracted data will be discussed, decisions documented, and when necessary, the authors

\section{Box 1 Search strategy by MEDLINE}

MEDLINE (via OVIDsp)

- exp Lipid Metabolism Disorders

- exp Body Fat Distribution

- lipoatrophy.mp.

- lipohypertrophy.mp.

- Lipodystrophy.mp.

- 1 or 2 or 3 or 4 or 5

- exp HIV Infection

- $\exp$ Anti-Retroviral Agents

- Nucleoside Reverse Transcriptase Inhibitors.mp.

- Non-Nucleoside reverse transcriptase inhibitors.mp.

- Protease Inhibitors.mp.

- Integrase Inhibitors.mp.

- Fusion Inhibitors.mp.

- Cor5.mp.

- 7 or 8 or 9 or 10 or 11 or 12 or 13 or 14.

6 and 15 . 
of the studies will be contacted for clarification. The reasons for excluding studies from the review will be documented. The following data will be extracted, checked and recorded.

- Characteristics of studies

Objective

Design

Year and country of study

Duration of study or follow-up

Source population

Sample size

Number of participants in each group monitoring

Losses to follow-up and/or reasons for nonparticipation (if applicable)

- Baseline characteristics of the study population Age

Age at the time of HIV infection

Number of naïve enrolled patients

Number of non-naïve enrolled patients

Duration of HIV infection

HIV risk factors

Log HIV RNA level

CD4-positive lymphocyte count

AIDS category (A, B, C)

Sex

Duration of ART

Type of current and prior ART

Fasting glucose level

Total cholesterol

Low density lipoprotein (LDL)

Triglycerides level

Metabolic syndrome

Family history of diabetes mellitus, hypertension, cardiovascular events and dyslipidaemia

Comorbidity (hepatitis C, tuberculosis, etc)

Consumption of alcohol, tobacco and other drugs

- Characteristics of lipodystrophy

Lipodystrophy definitions

Diagnostic criteria of lipodystrophy

Judgement of causality of lipodystrophy

Judgement of severity of lipodystrophy

Number of patients with lipodystrophy

Age at diagnosis of lipodystrophy

Occurrence of lipodystrophy according to antiretroviral regimen

Anthropometric characteristics (height, weight, body mass index, waist circumference, waist/hip ratio and percentage of body fat) at diagnosis of lipodystrophy.

\section{Assessment of risk of bias in included studies}

Owing to the absence of a validated instrument to assess the quality of observational studies of adverse drug reactions, there will be developed a checklist specifically for this review. This tool will be prepared based on the Guideline of the Cochrane Collaboration for nonrandomised studies. ${ }^{12}$ Two reviewers will assess independently the quality of eligible studies. Disagreements will be resolved by discussion and, when necessary, with the participation of a third reviewer.

\section{Measuring the effect}

The primary analysis will measure the frequency of occurrence of lipodystrophy, prevalence and incidence. The extraction of data of occurrence of the adverse reaction will be carried out in accordance with the study design. Thus, according to the methodological design of the included studies, it will be extracted measures of incidence or prevalence. Moreover, due to lack of standard diagnostic criteria for this adverse drug reaction, the different outcome measures reported in the included studies will be considered. Data on risk factors associated with lipodystrophy will also be extracted.

\section{Dealing with missing data}

Data assumed to be 'missing at random' may not be important and will be ignored. Data assumed to be 'not missing at random' will require contact with the original author to request the missing data and it will be carried out whenever possible.

\section{Assessment of heterogeneity and sensitivity analysis}

These analyses will be performed separately for each type of study design. Heterogeneity in the results will be graphically inspected and evaluated by means of $\chi^{2}$ test and $\mathrm{I}^{2}$ test. Sensitivity analysis will be explored through stratification by subgroups defined according to the type of outcome, diagnostic criteria and quality of studies.

\section{Risk assessment of publication bias}

The graphical funnel plot $^{17}$ will be used to investigate the presence of publication bias in the studies included in the review.

\section{Data synthesis and subgroup analysis}

We will present estimates on the occurrence, prevalence and incidence of lipodystrophy, according to study design, diagnosis criteria, type of outcome (lipoatrophy, lipohypertrophy and mixed form) and clinical and sociodemographic variables. A meta-analysis of the morbidity outcomes will be performed whenever appropriate. If applicable, the following subgroups will be analysed:

- Frequency of lipodystrophy according to duration of HIV infection;

- Frequency of lipodystrophy according to ART regimen;

- Frequency of lipodystrophy according to duration of ART;

- Frequency of lipodystrophy according to risk factors.

\section{DISSEMINATION}

The findings of this study will be disseminated through peer-reviewed publications and conference presentations. Updates of the review will be conducted to inform and guide healthcare practice. 


\section{DISCUSSION}

The toxicity of ART is a question of increasing relevance in the treatment of patients with HIV infection due to the need of maintaining ART indefinitely in order to achieve clinical benefits. ${ }^{18}$ Information on the occurrence of lipodystrophy among patients with HIV infection during current or previous use of ART is needed in order to support clinical and informed decision on HIV treatment and the use of ART. Systematic reviews only emphasising the benefit of drug therapies contribute to the omission of information on adverse effects of the interventions, thus preventing balanced decisions on benefits and risks. ${ }^{19}$

Knowledge regarding lipodystrophy among patients with HIV infection has increased since the beginning of the HAART era in the late 1990s. As a result, several randomised clinical trials and observational studies focusing on this subject have been published worldwide. However, the lack of a precise case definition of lipodystrophy and differences in the follow-up time of these studies has hampered the evaluation of morbidity estimates and risk factors associated with lipodystrophy. It is known that much of the evidence on adverse drug reactions comes from observational epidemiology studies due to the limitations of randomised clinical trials in evaluating this type of outcome. Notably, clinical trials are not adequate for detecting long-term outcomes (such as lipodystrophy), as they are, in general, of limited follow-up length. ${ }^{19}$

Some potential limitations of the study should be pointed out. Lipodystrophy does not have a precise case definition and a standardised diagnostic criterion. This being considered, our systematic review will evaluate observational studies investigating different outcome definitions and diagnostic methods. As a consequence, the review will include very heterogeneous studies, resulting in different groups of comparisons and more sophisticated analysis (eg, meta-analysis) may or may not be possible. Another potential limitation of our study is that mild to moderate cases of lipodystrophy may be underreported in the eligible studies, which could underestimate the frequency of lipodystrophy. Despite this, we believe that this study will contribute to the standardisation and better report of lipodystrophy related to HIV infection and ART.

In conclusion, compiled information on morbidity estimates and risk factors of lipodystrophy according to different groups of patients is needed and it will ease clinical decisions in relation to healthcare interventions for patients with HIV infection ,then contribute to lower morbidity and improve adherence to long-term ART

Contributors LGCL developed the methodological strategies under the guidance of DRGJ and participated in the drafting of the study protocol; DRGJ participated in the planning and drafting of the study protocol; EP participated in the planning and drafting of the study protocol; CMP conceived the study and drafted the study protocol. All the authors read and approved the final version of the study protocol for submission.
Funding This work was supported by the Universidade Federal de Minas Gerais (Pró-Reitoria de Pesquisa-PRPq/UFMG), Fundação de Amparo à Pesquisa do Estado Minas Gerais- FAPEMIG (Grant number APQ-02342-12) and Conselho Nacional de Desenvolvimento Científico e Tecnológico-CNPq (Grant number 484865/2011-0) A master's fellowship was awarded to LGCL by the FAPEMIG.

Competing interests None.

Provenance and peer review Not commissioned; externally peer reviewed.

Open Access This is an Open Access article distributed in accordance with the Creative Commons Attribution Non Commercial (CC BY-NC 3.0) license, which permits others to distribute, remix, adapt, build upon this work noncommercially, and license their derivative works on different terms, provided the original work is properly cited and the use is non-commercial. See: http:// creativecommons.org/licenses/by-nc/3.0/

\section{REFERENCES}

1. Bonfanti P, Ricci E, Landonio S, et al. Predictors of protease inhibitor-associated adverse events. Biomed Pharmacother 2001;55:321-3.

2. Bonolo PF, César CC, Acúrcio FA, et al. Non-adherence among patients initiating antiretroviral therapy: a challenge for health professionals in Brazil. AIDS 2005;19:S5-13.

3. Campos LN, César CC, Guimarães MCD. Quality of life among HIV/aids patients after initiating antiretroviral therapy. Clinics 2009;64:867-75.

4. Almeida LB, Giudici KV, Jaime PC. Consumo alimentar e dislipidemia decorrente da terapia antirretroviral combinada para infecção pelo HIV: uma revisão sistemática. Arq Bras Endocrinol Metabol 2009;53:519-27.

5. Menezes de Pádua CA, César CC, Bonolo PF, et al. High incidence of adverse reactions to initial antiretroviral therapy in Brazil. Braz $J$ Med Biol Res 2006;39:495-505.

6. Han SH, Zhou J, Saghayam S, et al. Prevalence of and risk factors for lipodystrophy among HIVinfected patients receiving combined antiretroviral treatment in the Asia-Pacific region: results from the TREAT Asia HIV Observational Database (TAHOD). Endocr J 2011;58:475-84.

7. Ammassari A, Antinori A, Cozzi-Lepri A, et al. Relationship between HAART adherence and adipose tissue alterations. J Acquir Immune Defic Syndr 2002;31:S140-4.

8. Carr A. HIV lipodystrophy: risk factors, pathogenesis, diagnosis and management. AIDS. 2003;17:S141-148.

9. Wohl DA. Diagnosis and management of body morphology changes and lipid abnormalities associated with HIV Infection and its therapies. Top HIV Med 2004;12:89-93.

10. Diehl LA, Dias JR, Paes AC, et al. Prevalence of HIV-associated lipodystrophy in Brazilian outpatients: relation with metabolic syndrome and cardiovascular risk factors. Arq Bras Endocrinol Metabol 2008;52:658-67.

11. de Waal R, Cohen K, Maartens G. Systematic review of antiretroviral-associated lipodystrophy: lipoatrophy, but not central fat gain, is an antiretroviral adverse drug reaction. PLOS ONE 2013;8:63623.

12. Chen D, Misra A, Garg A. Lipodystrophy in Human Immunodeficiency Virus-Infected Patients. J Clin Endocrinol Metab 2002;87:4845-56.

13. Carr A, Emery S, Law M, et al. An objective case definition of lipodystrophy in HIV-infected adults: a case-control study. Lancet 2003;361:726-35.

14. Grinsponn S, Carr A. Cardiovascular risk and body-fat abnormalities in HIV-infected adults. N Engl J Med 2005;352:48-62.

15. University of York. Centre for reviews and dissemination. Systematic reviews: CRD's guidance for undertaking reviews in health care. Centre for Reviews and Dissemination, 2009.

16. Higgins JPT, Green S, eds. Cochrane Handbook for systematic reviews of interventions version 5.1.0 [updated March 2011]. The Cochrane Collaboration, 2011.

17. Egger M, Davey Smith D, Schneider M, et al. Bias in meta-analysis detected by a simple, graphical test. BMJ 1997;315:629-34.

18. Carr A, Cooper DA. Adverse effects of antiretroviral therapy. Lancet 2000;356:1423-30.

19. Loke YK, Price D, Herxheimer A. Systematic reviews of adverse effects: framework for a structured approach. BMC Med Res Methodol 2007;7:32. 